\title{
Current status of circulating tumor cell androgen receptor splice variant-7 in metastatic castration-resistant prostate cancer
}

\author{
Shinichi Sakamoto \\ Department of Urology, Chiba University Graduate School of Medicine, Chiba, Japan \\ Correspondence to: Shinichi Sakamoto. Associate Professor, Department of Urology, Chiba University Graduate School of Medicine, 1-8-1 Inohana, \\ Chuo-ku, Chiba 260-8670, Japan. Email: rbatbat1@gmail.com. \\ Provenance: This is an invited article commissioned by the Editorial Board member Dr. Xiao Li, MD (Department of Urology, Jiangsu Cancer \\ Hospital \& Jiangsu Institute of Cancer Research \& Nanjing Medical University Affiliated Cancer Hospital, Nanjing, China). \\ Comment on: Sharp A, Welti JC, Lambros MBK, et al. Clinical utility of circulating tumour cell androgen receptor splice variant-7 status in metastatic \\ castration-resistant prostate cancer. Eur Urol 2019;76:676-85.
}

Submitted Nov 04, 2019. Accepted for publication Dec 24, 2019.

doi: 10.21037/atm.2019.12.137

View this article at: http://dx.doi.org/10.21037/atm.2019.12.137

In castration resistant prostate cancer (CRPC), the androgen receptor $(\mathrm{AR})$ pathway remains activated through upregulation of AR, mutation, and paracrine/autocrine androgen synthesis (1). Clinical significance of AR variant 7 (AR-V7) in circulating tumor cells (CTCs) was first reported by Antonarakis et al., which demonstrated a strong association with abiraterone and enzalutamide treatment resistance (2). On the other hand, due to the naïve nature of CTCs, detection of AR-V7 status by CTCs had some limitations includes reproducibility of AR-V7 positivity, heterogeneity between institutions. Furthermore, previous studies rarely attempt to compare the AR-V7 expression between tissue and CTCs. There have been several issues to be solved regarding the analysis of AR-V7 in CTCs.

In order to answer those questions, Sharp et al. conducted a multi-institutional study to prove the reproducibility as well as the clinical significance of AR-V7 in CTCs. They studied CTC AR-V7 status in 227 peripheral blood samples, from 181 metastatic CRPC (mCRPC) patients with CTC counts and matched mCRPC biopsies. They used AdonaTest for AR-V7 mRNA detection, and CellSearch systems for the CTC counts. Intralaboratory reproducibility was $92 \%$ between replicate. Between institutions, the overall interlaboratory agreement between London, UK, and JHU in Baltimore, USA, was as high as $86 \%$. Among all cohorts, $35 \%$ of samples showed CTC+/AR-V7+. They demonstrated that CTC+/AR-V7+ samples had higher CellSearch CTC counts and biopsy AR-V7 protein expression than CTC+/AR-V7- samples.
There was a positive correlation between AR-V7 mRNA expression and CTC counts. CTC+/AR-V7+ showed the worst survival, followed by CTC+/AR-V7-. On the other hand, CTC- showed the most favorable prognosis.

Another unique aspect of the article may be that they conducted a multivariate analysis of CTC AR-V7 and CellSearch CTC counts together with other clinical factors. Previous reports mainly looked at the role of AR-V7 status in CTCs in a univariate manner $(2,3)$. Data demonstrated prognostic significance of CTC AR-V7 and CellSearch CTC counts $(\geq 5)$ over the common clinical factors include prostate-specific antigen (PSA), lactate dehydrogenase $(\mathrm{LDH})$, and even the presence of visceral metastasis. As Prostate Cancer Clinical Trials Working Group 3 (PCWG3) recommended serial biologic profiling using tumor samples from biopsies and blood-based diagnostics (4), current data confirmed the clinical significance of biological profiling through liquid biopsies.

Compare to recent published phase III clinical trial (ARMOR3-SV) using the same AdonaTest for detecting CTC ARV-7 (5), current article showed relatively high AR$\mathrm{V} 7$ prevalence. AR-V7 prevalence was $8 \%$ in ARMOR3SV, while $35 \%$ in the present study. The reason may be explained by the difference in patients' backgrounds. Median PSA in ARMOR3-SV trial was $72.0 \mathrm{ng} / \mathrm{mL}$ in enzalutamide arm and $96.15 \mathrm{ng} / \mathrm{mL}$ in galeterone arm. However, in the current study, the median PSA was $>100 \mathrm{ng} / \mathrm{mL}$ among all the groups. It was also described that AR-V7+ was observed in $15 \%$ of patients with PSA 
$>31 \mathrm{ng} / \mathrm{mL}$ and $30 \%$ with $>21$ bone lesions in ARMOR3SV (5). The heterogeneity observed between studies may be explained by the metastatic burden of prostate cancer patients enrolled in the studies.

In terms of detecting AR-V7, two major approaches are available, such as AdonaTest CTC AR-V7 mRNA assay and Epic Sciences (Epic; San Diego, CA, USA) CTC nuclear-specific AR-V7 protein assay. AdonaTest was first demonstrated by Antonarakis et al., while CTC nuclearspecific AR-V7 assay was utilized by Scher et al. $(2,6)$. Since heterogeneity of AR-V7 positivity between two tests and among institutions has been an issue, prospective multicenter validation of AR-V7 study (the PROPHECY study) was conducted. Twenty-four percent of samples were AR-V7 positive by the JHU mRNA assay, while $9 \%$ of samples were AR-V7 positive by the Epic protein-based assay. Clinical significance of AR-V7 status by two assays were similar among patients treated with Enzalutamide or Abiraterone (7).

One of the critical characteristics of AR-V7 may be the association between AR amplification. Previous reports demonstrated an overlapped expression pattern of AR-V7 and full-length AR (AR-FL) among CRPC patients (8). Compare to the primary tumor, AR-V7 increased 53 folds, while AR-FL also increased ten folds in CRPC (8). In biological aspects, AR-V7 was generated through RNA splicing factors. Under androgen deprivation therapy, the recruitment of several RNA splicing factors to the 3' splicing site for AR-V7 was increased. RNA splicing enhancers and their binding proteins (U2AF65 and ASF/ SF2) play a critical role in splicing AR pre-mRNA into AR-V7 (9). In this aspect, the presence of AR-FL and AR-V7 may be a part of a common pathway. Del Re et al. investigated AR-FL and AR-V7 in association with resistance to the AR-directed therapy. Among 73 CRPC patients, AR-FL was detected in all patients, while AR-V7 was detected in $22 \%$ of patients. AR-FL expression was significantly higher in AR-V7+ patients compare to AR-V7patients. There was a significant correlation between ARFL and AR-V7 expression ( $r=0.581, \mathrm{P}<0.0001)$. Presence of AR-V7 as well as high AR-FL copy numbers associated with poor prognosis (10).

Based on the next-generation sequence of biopsy samples of metastatic tissues, the detection of AR-V7 was 10\% among CRPC patients (11). Among AR splicing variants, besides $A R-V 7$, other variants exist, such as $A R-$ $\mathrm{V} 1, \mathrm{~V} 3$, and v567es (11). Tagawa et al. investigated the prognostic significance of AR-FL, AR-V7, and AR-567es in CTCs from patients receiving docetaxel or cabazitaxel in TAXYNERGY (NCT01718353) study. Of those CTCs were detected, $51.9 \%$ of the samples were CTC+/AR-V7+, and $26.9 \%$ were CTC+/AR-567es+. Presence of AR-V7 or AR-567es related to shorter PFS even among patients treated with taxane-based therapy. PSA response rate $(>50 \%)$ was very similar between AR-V7+ $(58 \%)$ and AR567 es+ $(57 \%)$ patients. They concluded that the presence of AR-V7 might confer modest taxane resistance, although significantly less than that on AR targeted drugs (12).

Recently, Cucchiara et al. reported an interesting finding related to a gonadotropin-releasing hormone (GnRH) receptor antagonist. In mice bearing $\mathrm{AR}-\mathrm{V} 7$ positive $\mathrm{VCaP}$ xenograft tumors, the size of tumors in degarelix-treated mice were $67 \%$ of those in the leuprolide-treatment group. Measurements of intratumoral steroids by LC/MS identified that degarelix significantly decreased the testosterone level and steroidogenesis pathway intermediates, comparable to surgical castration or leuprolide. The author described the potential clinical advantage of degarelix over GHRH agonists in AR-V7 harboring tumors (13).

A series of mechanisms exist during the development of CRPC. Resistance mechanisms independent of AR$\mathrm{V} 7$ include AR gain or mutations, alternative AR variants, lineage plasticity, genomic structural rearrangements, and AR enhancer amplification $(1,7)$. Furthermore, besides AR, compound genomic alterations of TP53, PTEN, and RB1 tumor suppressors are related to aggressive variant prostate cancer (AVPC) (14). The St Gallen Advanced Prostate Cancer Consensus Conference 2017 reported that the mutation in DNA damage response (DDR) genes, such as BRCA1, BRCA2, and ATM, should be reported in metastatic prostate cancer because that knowledge will likely influence management decisions (15). Treatment with the PARP inhibitor olaparib showed a high response rate $(88 \%)$ in patients who had a homozygous deletion, deleterious mutations, or both in DNA repair genes, even after the development of resistance against the standard treatment (16). To investigate alteration in multiple target genes, it may be a practical approach to utilize the cfDNA in addition to CTCs (17).

As precision medicine is on the rise, the genomic analysis of individual patients is becoming more and more critical. As a series of retrospective and prospective trial identified, it is no doubt that the presence of AR-V7 in CTCs is related to treatment resistance to AR targeted therapy. However, alteration in tumor suppressor and DDR related genes are also involved during the development of CRPC $(14,15)$. It may be the way to combine AR-V7 status in CTCs together 
with the mutational landscape of $\mathrm{AR}$ and other prognostic genes in cfDNA to determine the optimal treatment sequence in CRPC patients. Based on biological profiling through the liquid biopsy, risk classification, and treatment strategy of metastatic prostate cancer may drastically change in the near future.

\section{Acknowledgments}

None

\section{Footnote}

Conflicts of Interest: The author has no conflicts of interest to declare.

Ethical Statement: The author is accountable for all aspects of the work in ensuring that questions related to the accuracy or integrity of any part of the work are appropriately investigated and resolved.

\section{References}

1. Annala M, Vandekerkhove G, Khalaf D, et al. Circulating tumor DNA genomics correlate with resistance to abiraterone and enzalutamide in prostate cancer. Cancer Discov 2018;8:444-57.

2. Antonarakis ES, Lu C, Wang H, et al. AR-V7 and resistance to enzalutamide and abiraterone in prostate cancer. N Engl J Med 2014;371:1028-38.

3. Antonarakis ES, Lu C, Luber B, et al. Clinical significance of androgen receptor splice variant-7 mRNA detection in circulating tumor cells of men with metastatic castrationresistant prostate cancer treated with first- and second-line abiraterone and enzalutamide. J Clin Oncol 2017;35:2149-56.

4. Scher HI, Morris MJ, Stadler WM, et al. Trial design and objectives for castration-resistant prostate cancer: updated recommendations from the Prostate Cancer Clinical Trials Working Group 3. J Clin Oncol 2016;34:1402-18.

5. Taplin ME, Antonarakis ES, Ferrante KJ, et al. Androgen receptor modulation optimized for response-splice variant: a phase 3 , randomized trial of galeterone versus enzalutamide in androgen receptor splice variant-7expressing metastatic castration-resistant prostate cancer. Eur Urol 2019;76:843-51.

6. Scher HI, Lu D, Schreiber NA, et al. Association of ARV7 on circulating tumor cells as a treatment-specific biomarker with outcomes and survival in castration- resistant prostate cancer. JAMA Oncol 2016;2:1441-9.

7. Armstrong AJ, Halabi S, Luo J, et al. Prospective multicenter validation of androgen receptor splice variant 7 and hormone therapy resistance in high-risk castrationresistant prostate cancer: the PROPHECY study. J Clin Oncol 2019;37:1120-9.

8. Yu Z, Chen S, Sowalsky AG, et al. Rapid induction of androgen receptor splice variants by androgen deprivation in prostate cancer. Clin Cancer Res 2014;20:1590-600.

9. Liu LL, Xie N, Sun S, et al. Mechanisms of the androgen receptor splicing in prostate cancer cells. Oncogene 2014;33:3140-50.

10. Del Re M, Crucitta S, Sbrana A, et al. AR-V7 and ARFL expression is associated with clinical outcome: a translational study in patients with castrate resistant prostate cancer. BJU Int 2019. [Epub ahead of print].

11. Robinson D, Van Allen EM, Wu YM, et al. Integrative clinical genomics of advanced prostate cancer. Cell 2015;161:1215-28.

12. Tagawa ST, Antonarakis ES, Gjyrezi A, et al. Expression of AR-V7 and ARv567es in circulating tumor cells correlates with outcomes to taxane therapy in men with metastatic prostate cancer treated in TAXYNERGY. Clin Cancer Res 2019;25:1880-8.

13. Cucchiara V, Yang JC, Liu C, et al. GnRH antagonists have direct inhibitory effects on castration-resistant prostate cancer via intracrine androgen and AR-V7 expression. Mol Cancer Ther 2019;18:1811-21.

14. Hamid AA, Gray KP, Shaw G, et al. Compound genomic alterations of TP53, PTEN, and RB1 tumor suppressors in localized and metastatic prostate cancer. Eur Urol 2019;76:89-97.

15. Gillessen S, Attard G, Beer TM, et al. Management of patients with advanced prostate cancer: the report of the Advanced Prostate Cancer Consensus Conference APCCC 2017. Eur Urol 2018;73:178-211.

16. Mateo J, Carreira S, Sandhu S, et al. DNA-Repair defects and olaparib in metastatic prostate cancer. N Engl J Med 2015;373:1697-708.

17. Vandekerkhove G, Struss WJ, Annala M, et al. Circulating tumor DNA abundance and potential utility in de novo metastatic prostate cancer. Eur Urol 2019;75:667-75.

Cite this article as: Sakamoto S. Current status of circulating tumor cell androgen receptor splice variant-7 in metastatic castration-resistant prostate cancer. Ann Transl Med 2019;7(Suppl 8):S375. doi: 10.21037/atm.2019.12.137 